\title{
Gengivectomia com finalidade estética: relato de dois casos clínicos
}

\author{
Aesthetical gengivectomy: reporto of two clinical cases \\ Gengivectomía estética: informe de dos casos clínicos
}

\begin{abstract}
Cláudio Roberto Tavares Pereira Filho1, Sara Maria Rodrigues de Sousa1, Larice Kércia Braz Monteiro ${ }^{1,2}$, Vilana Maria Adriano Araújo ${ }^{1,2,3}$, Flávia Jucá Alencar e Silva ${ }^{1,2}$, Ernanda Maria de Araújo Sales $^{1,2}$, Mariana Canuto Melo de Sousa Lopes ${ }^{1,2}$, Breno Souza Benevides ${ }^{2}$, João Eudes Teixeira Pinho Filho ${ }^{2}$, Mariana Vasconcelos Guimarães ${ }^{1,2,3, *}$.
\end{abstract}

\section{RESUMO}

Objetivo: Discutir os resultados do procedimento de aumento de coroa clínica com finalidade estética, apresentando dois casos clínicos de pacientes com hiperplasia gengival e erupção dental passiva alterada. Detalhamento do Caso: Ambos os pacientes compareceram ao Complexo Odontológico São João Calábria da UNICATÓLICA queixando-se da estética de seus sorrisos. Após os exames, constatou-se saúde periodontal, porém havia notória relação desarmônica entre o comprimento e o formato dos dentes, bem como alta exposição de tecido gengival ao sorrir. Foram realizadas sondagem transulcular, fotografias e exame radiográfico. Os pacientes assinaram o Termo de Consentimento Livre e Esclarecido. No caso da erupção passiva alterada, optou-se por bisel interno sem descolamento de retalho seguido do refinamento das margens gengivais com tesoura Castroviejo. No caso de hiperplasia gengival, a técnica escolhida foi de bisel externo junto com o procedimento de peeling gengival seguido da frenectomia labial superior. Os dois pacientes foram acompanhados pelo período de 7,14, 90 e 150 dias, não apresentaram queixas estéticas e se mostraram satisfeitos com o resultado final. Considerações finais: Para a realização da gengivectomia vários fatores devem ser analisados previamente ao procedimento, entre eles fatores estéticos, biótipo periodontal, a causa da alteração e a necessidade ou não de osteotomia.

Palavras-chave: Periodontia, Hiperplasia gengival, Estética.

\begin{abstract}
Objective: To discuss the results of the aesthetic purpose clinical crown augmentation procedure, presenting two clinical cases of patients with gingival hyperplasia and altered passive dental eruption. Case details: Both patients attended the UNICATÓLICA São João Calabria Dental Complex complaining about the aesthetics of their smiles. After the exams, periodontal health was found, but there was a notorious disharmonic relationship between the length and shape of the teeth, as well as high exposure of gum tissue when smiling. Transulcular probing, photographs and radiographic examination were performed. The patients signed the Informed Consent Form. In the case of altered passive eruption, an internal bevel without flap detachment was chosen, followed by refinement of the gingival margins with Castroviejo scissors. In the case of gingival hyperplasia, the technique chosen was external bevel along with the gingival peeling procedure followed by upper lip frenectomy. The two patients were followed for a period of 7.14, 90 and 150 days, had no aesthetic complaints and were satisfied with the final result. Final considerations: In order to perform gingivectomy, several factors

${ }^{1}$ Centro Universitário Católica de Quixadá (UNICATÓLICA), Quixadá-CE.

2 Programa de Pós-Graduação em Odontologia da Universidade Federal do Ceará (UFC), Fortaleza-CE.

3 Programa de Pós-Graduação em Farmacologia da Universidade Federal do Ceará (UFC), Fortaleza-CE.

*E-mail:marianav_guimaraes@yahoo.com.br
\end{abstract}


must be analyzed prior to the procedure, including aesthetic factors, periodontal biotype, the cause of the alteration and the need or not for osteotomy.

Keywords: Periodontics, Gingival hyperplasia, Aesthetics.

\section{RESUMEN}

Objetivo: Discutir los resultados del procedimiento de aumento clínico de la corona con fines estéticos, presentando dos casos clínicos de pacientes con hiperplasia gingival y erupción dental pasiva alterada. Detalles del caso: Ambos pacientes asistieron al Complejo Dental UNICATÓLICA São João Calabria quejándose de la estética de sus sonrisas. Después de los exámenes, se encontró salud periodontal, pero hubo una notoria relación disarmónica entre la longitud y la forma de los dientes, así como una alta exposición del tejido de las encías al sonreír. Se realizaron sondeos transulculares, fotografías y examen radiográfico. Los pacientes firmaron el formulario de consentimiento informado. En el caso de erupción pasiva alterada, se eligió un bisel interno sin desprendimiento de la aleta, seguido por el refinamiento de los márgenes gingivales con tijeras de Castroviejo. En el caso de la hiperplasia gingival, la técnica elegida fue el bisel externo junto con el procedimiento de exfoliación gingival seguido de la frenectomía del labio superior. Los dos pacientes fueron seguidos por un período de 7.14, 90 y 150 días, no tuvieron quejas estéticas y quedaron satisfechos con el resultado final. Consideraciones finales: para realizar la gingivectomía, se deben analizar varios factores antes del procedimiento, incluidos los factores estéticos, el biotipo periodontal, la causa de la alteración y la necesidad o no de osteotomía.

Palabras clave: Periodoncia, Hiperplasia gingival, Estética.

\section{INTRODUÇÃO}

O sorriso estético é um objetivo bastante almejado na sociedade contemporânea. Além de fatores inerentes às estruturas dentárias, sabe-se que fatores relacionados aos lábios e, especialmente, aos tecidos periodontais são imprescindíveis na obtenção da harmonia estética do sorriso. De fato, a estética periodontal, ou simplesmente estética vermelha, representada pela gengiva, tem sido cada vez mais valorizada (BATRA $\mathrm{P}$, et al., 2018).

Um dos mais frequentes fatores relacionados a alterações do contorno gengival e que consequentemente interfere com a estética do sorriso é a excessiva exposição gengival ao sorrir, denominado, popularmente, como sorriso gengival (SHAHABE SA, et al., 2012). Existem várias causas associadas a tal problema estético, como a hiperatividade do músculo elevador do lábio superior, o crescimento vertical da maxila, o aumento do tecido gengival propriamente dito e ou a erupção passiva alterada. Há ainda fatores que levam a hiperplasia gengival de origem neoplásica, hereditária, medicamentosa, inflamatória ou associação de duas ou mais. Além disso, outros fatores locais, como má posição dental, presença de cárie e uso de dispositivos ortodônticos podem levar a um aumento gengival de origem inflamatória, o que não é incomum (RIBEIRO FV, et al., 2014; KALSI HJ, et al., 2015).

Procedimentos cirúrgicos periodontais têm ganhado destaque por sua variedade de técnicas cirúrgicas que possibilitam não apenas a boa função dos tecidos periodontais, mas também sua estética (SILVA CO, et al., 2015). Nesse contexto, a gengivoplastia consiste em uma remodelação cirúrgica do tecido gengival e papilas para correção de deformidades, com objetivo a estética. Dentre seus procedimentos, enquadra-se a gengivectomia como uma técnica de Aumento de Coroa Clínica (ACC), a qual é, muitas vezes, realizada com finalidade estética (MALKINSON S, et al., 2013; SMITH MM, 2013; KALSI HJ, et al., 2015).

O ACC através de gengivectomia é um dos procedimentos cirúrgicos periodontais mais frequentemente realizados na prática clínica odontológica e consiste em reduzir o tecido gengival excessivo, expor as coroas clínicas desejáveis e, quando necessário, restabelecer a distância biológica apropriada. Assim, a finalidade deste procedimento pode ser funcional, quando, por exemplo, margens cervicais de lesões cariosas ou 
fraturas ocorrem em nível subgengival, ou estética, quando prevalece a busca por um sorriso mais agradável visualmente por redução da quantidade de gengiva recobrindo o dente ou simplesmente restabelecimento anatômico favorável do contorno gengival (SEIXAS MR, et al., 2011).

A técnica cirúrgica escolhida para ambas às situações depende de vários fatores que determinam a escolha de incisões e a necessidade ou não de osteotomia e, em casos de finalidade estética, vários padrões estéticos cientificamente pré-estabelecidos devem ser levados em consideração (RIBEIRO FV, et al., 2014; FALABELLA MEV, et al., 2015; BENNANI V, et al., 2017).

Diante disso, o presente estudo objetiva a apresentação do manejo cirúrgico e dos resultados clínicos de dois procedimentos de um ACC em pacientes com insatisfação na estética do sorriso. Esses dois casos foram escolhidos por serem de etiologias frequentes de sorriso gengival, especificamente Erupção Dental Passiva Alterada e Hiperplasia Gengival, e pela possibilidade de abordagens diferenciadas para ambos os casos entre si.

\section{DETALHAMENTO DO CASO}

Este estudo foi aprovado pelo Comitê de Ética do Centro Universitário Católica de Quixadá UNICATÓLICA (protocolo 3.601.152) e teve autorização do local onde foi realizado o procedimento. Os pacientes envolvidos receberam todas as informações éticas necessárias e assinaram o termo de confidencialidade dos dados e de consentimento livre e esclarecido previamente a realização dos referidos procedimentos cirúrgicos.

Durante a anamnese, os pacientes afirmaram não serem fumante e apresentarem boa saúde, sem qualquer comprometimento sistêmico ou uso de medicação contínua. Ao exame clínico intraoral, foi verificado que os índices de placa visível e de sangramento gengival de ambos os pacientes estavam compatíveis com saúde periodontal, porém havia notória relação desarmônica entre o comprimento e o formato dos dentes quanto à exposição de tecido gengival ao sorrir. As análises do tecido gengival e ósseo foram realizadas através de sondagem transulcular, fotografias e exame radiográfico panorâmico.

\section{Caso clínico 1}

Paciente S.M.R. do sexo feminino, 21 anos, compareceu à Clínica do Complexo Odontológico São João da Calábria, do Centro Universitário Católica de Quixadá, Ceará, Brasil, com queixa principal de sorriso gengival e "dentes pequenos". Em repouso e ao sorriso máximo, foi constatada a necessidade de ACC na arcada superior, abrangendo os elementos 13 a 23.

Apesar da excessiva exposição de gengiva ao sorrir (Figura 1A), ao exame clínico de sondagem transulcular e ao exame radiográfico (Figura 1B), não se observou necessidade de osteotomia para acomodação da inserção tecidual supracrestal, uma vez que a distância da junção cemento esmalte à crista óssea alveolar observada foi de 2 a $3 \mathrm{~mm}$. Considerando que a exposição de gengiva ao sorrir não estava relacionada às causas de proliferação intensa de tecido gengival ou alterações ósseas e/ou musculares, constatou-se o diagnóstico de erupção dental passiva alterada do tipo $1 \mathrm{~A}$ na classificação de Coslet (1977) (YADAV SR, et al., 2015).

Optou-se pela realização do procedimento através de técnica baseada em Flapless, consistindo em bisel interno sem posterior descolamento de retalho. Procedeu-se, então, com anestesia infiltrativa com Lidocaína $2 \%$ 1:100.000 (DFL, Brasil), logo em seguida foram demarcados os futuros zênites com a sonda Carolina do

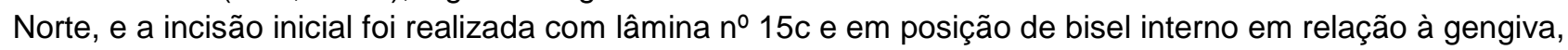
desenhando um colarinho gengival primeiramente do lado direito da paciente (Figura 1C), correspondente aos elementos $11,12,13$, e em seguida do lado esquerdo, correspondente aos elementos 21, 22, 23, seguido da incisão intrasulcular e remoção do colarinho com a Cureta Crane Kaplan.

Posteriormente, procedeu-se com a realização de um refinamento das margens gengivais com tesoura Castroviejo, removendo rebarbas (Figura 1D)._Por fim, foi colocado cimento cirúrgico sobre a área operada e instruído ao paciente o bochecho de digluconato de clorexidina $0,12 \%$ e prescrição medicamentosa para 0 controle da dor com Dipirona $500 \mathrm{mg}$. A paciente foi reavaliada em 7,14, 90 e 150 dias, e não apresentou queixas estéticas e se mostrou muito satisfeita com o resultado final (Figura 2). 
Figura 1 - Imagens antes, durante e após o procedimento de Gengivectomia da paciente S.M.R
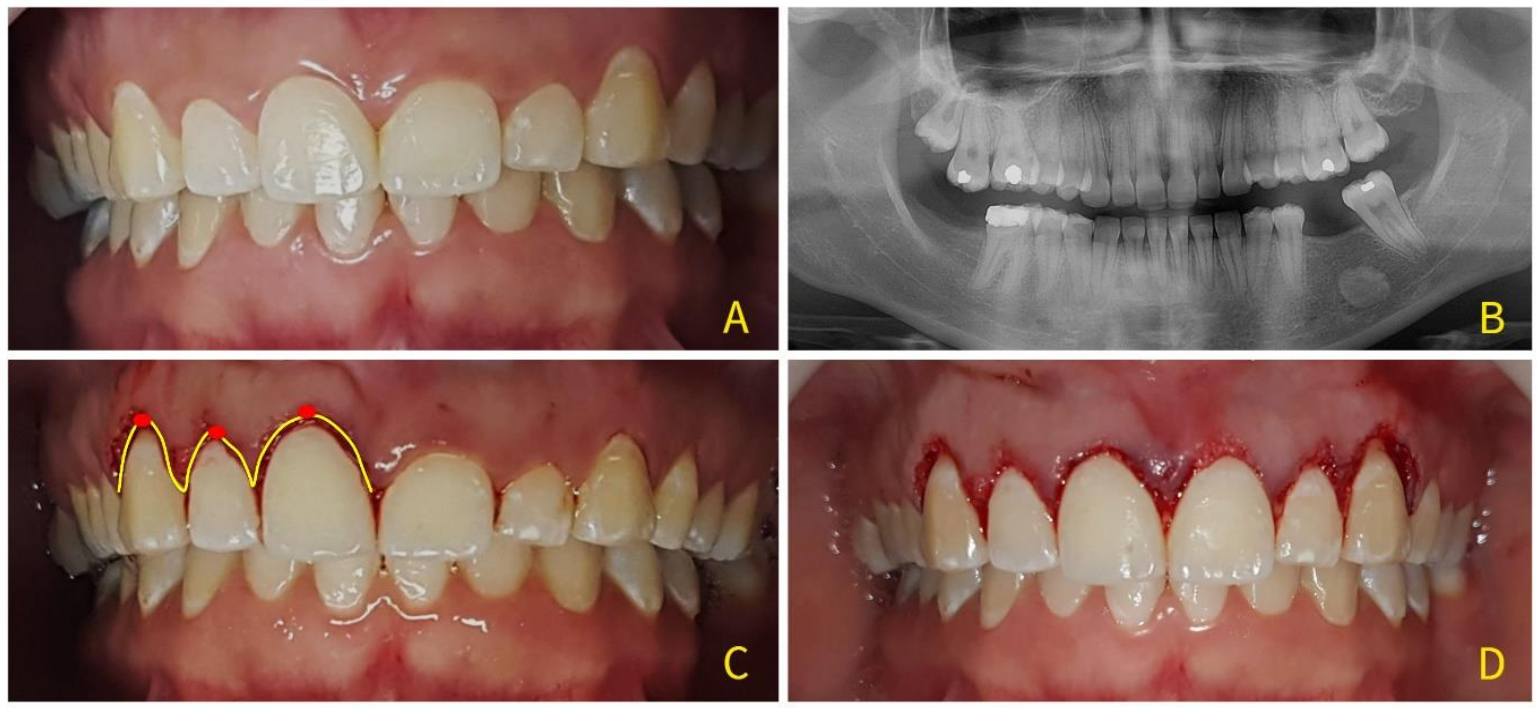

Legenda: (A) Aspecto inicial da paciente S.M.R. (B) Radiografia Panorâmica. (C) Transcirurgico. (D) Final pré cimento cirúrgico. Fonte: Guimarães MV, et al., 2020.

Figura 2 - Comparativo antes e após o procedimento de Gengivectomia da paciente S.M.R.

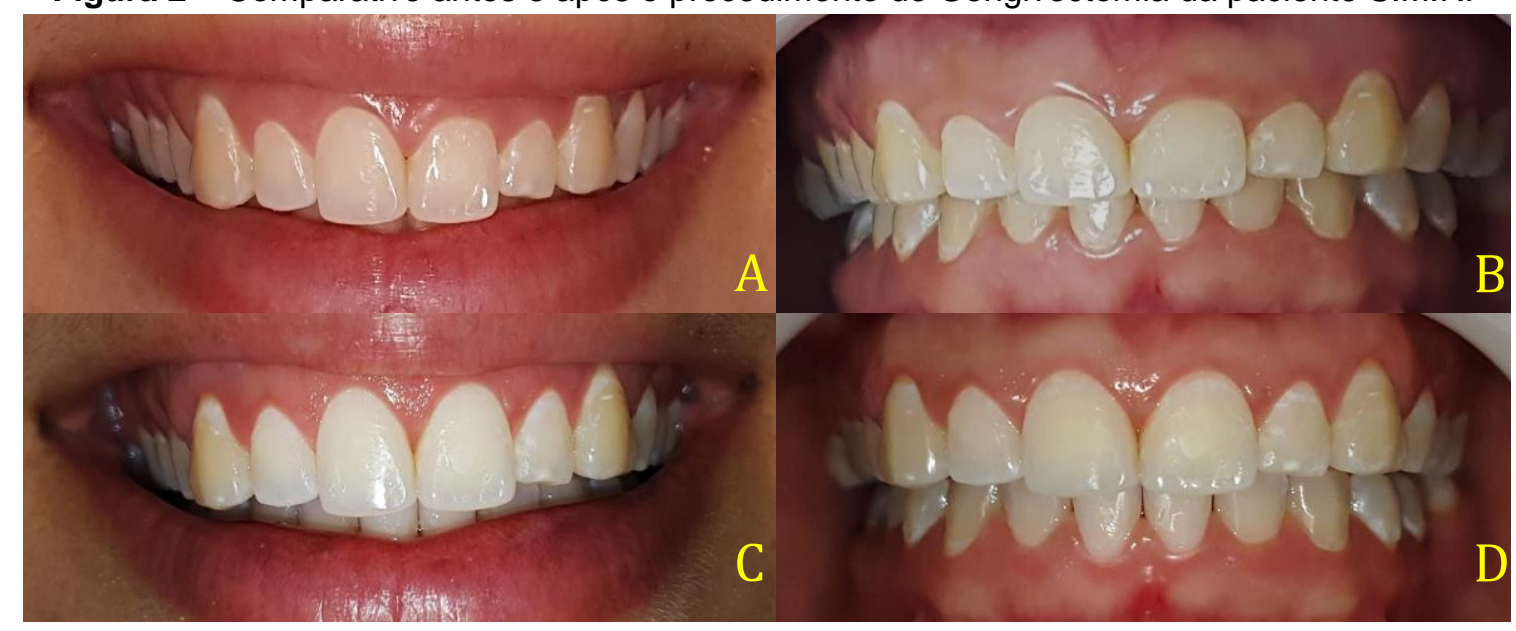

Legenda: (A e B) Aspecto inicial da paciente S.M.R.

(C e D) Aspecto final após 150 dias.

Fonte: Guimarães MV, et al., 2020.

\section{Caso clínico 2}

Paciente F.A.M do sexo masculino, 24 anos, compareceu à Clínica do Complexo Odontológico São João da Calábria, do Centro Universitário Católica de Quixadá, Ceará, Brasil, com queixa principal de "uma grande quantidade de gengiva aparente e os dentes muito pequenos". Em repouso e ao sorriso máximo, foi constatada a necessidade de ACC na arcada superior, abrangendo os elementos 14 a 24.

Constatou-se hiperplasia gengival (Figura 3A), com profundidade de sondagem de até $5 \mathrm{~mm}$. Ao exame radiográfico panorâmico (Figura 3B), entretanto, não foram observadas alterações ósseas, indicando a presença de pseudobolsas. Assim optou-se pela realização do aumento de coroa através de incisão em bisel externo seguida de procedimento de peeling gengival, uma vez que havia necessidade de redução da espessura de tecido gengival e remoção de suas manchas melânicas.

O procedimento iniciou-se com anestesia infiltrativa com Lidocaína 2\% 1:100.000 (DFL, Brasil) e demarcação dos futuros zênites com a sonda Carolina do Norte. A incisão inicial foi realizada com Gengivótomo de Kirkland em posição de bisel externo a 45ํe em relação ao dente, desenhando um colarinho, 
o qual foi seguidamente removido com Cureta Crane Kaplan (Figura 3D), realização de peeling gengival e um refinamento das margens gengivais com tesoura Castroviejo. Observou-se que a posição do freio labial superior poderia comprometer o resultado final, então foi realizado frenectomia labial superior e suturas interrompidas simples com fios de sutura. Por fim foi colocado cimento cirúrgico sobre a área operada e instruído ao paciente o uso de digluconato de clorexidina $0,12 \%$ e prescrição medicamentosa para o controle da dor com Dipirona $500 \mathrm{mg}$. O paciente foi reavaliada em 7,14, 90 e 150 dias, não apresentou queixas estéticas e se mostrou muito satisfeito com o resultado final.

Figura 3 - Imagens antes, durante e após o procedimento de Gengivectomia do paciente F.A.M.
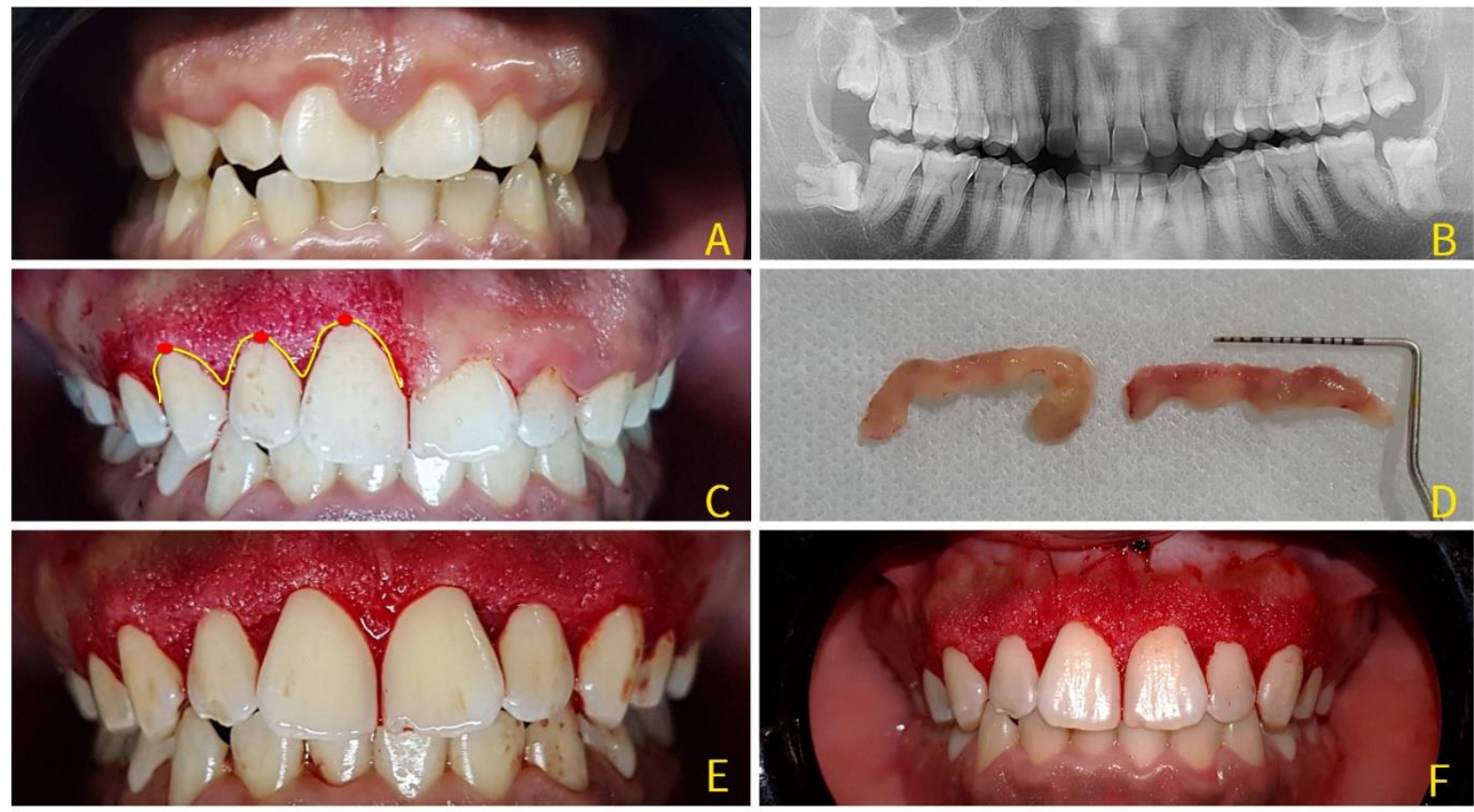

Legenda: (A) Aspecto inicial do paciente F.A.M. (B) Radiografia Panorâmica. (C) Transcirurgico. (D) Colarinhos gengivais removidos. (E e F) Aspecto pós-cirúrgico.

Fonte: Guimarães MV, et al., 2020.

Figura 4 - Comparativo antes e após o procedimento de Gengivectomia do paciente F.A.M.
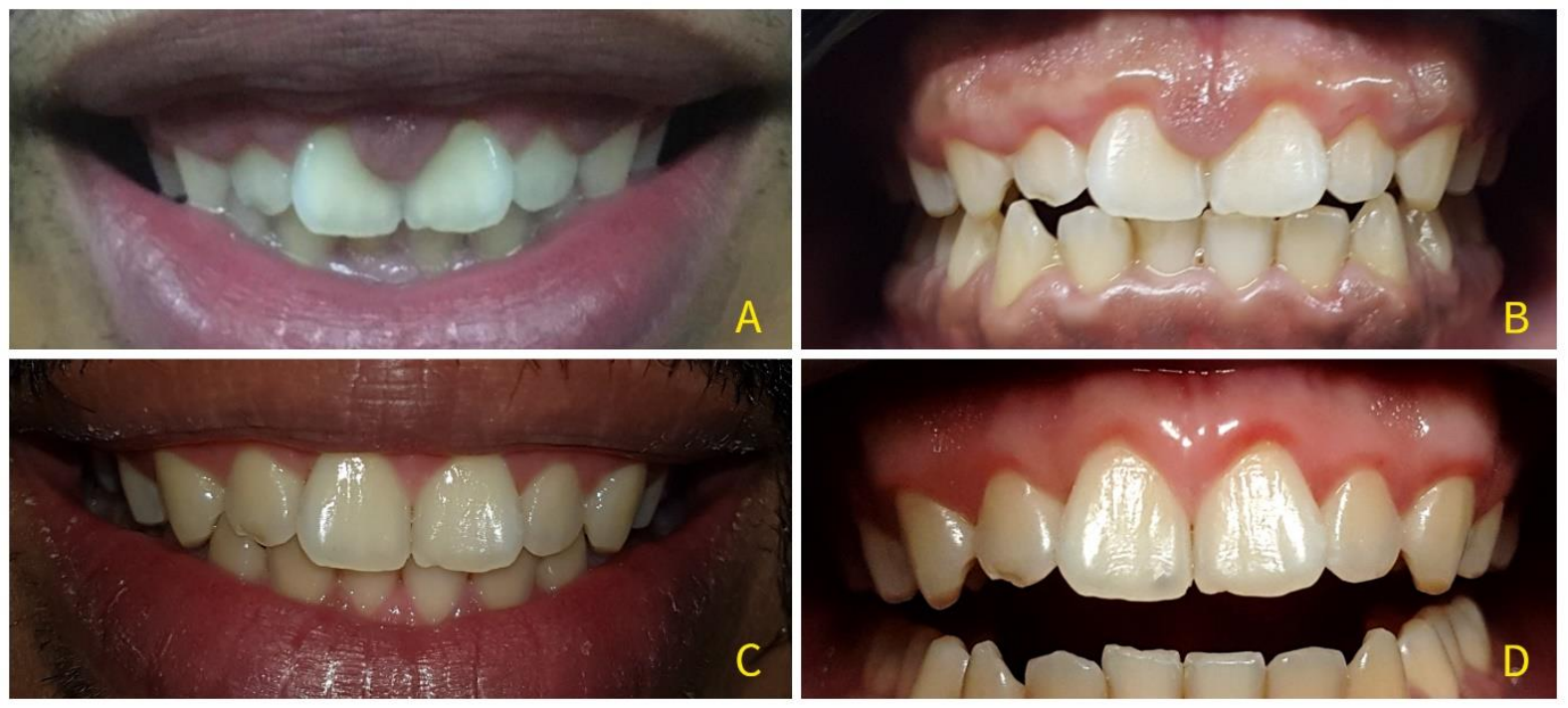

Legenda: (A e B) Aspecto inicial da paciente F.A.M. (C e D) Aspecto final após 150 dias.

Fonte: Guimarães MV, et al., 2020. 


\section{DISCUSSÃO}

A gengivectomia é um dos procedimentos cirúrgicos periodontais mais frequentemente realizados na prática clínica odontológica e consiste em reduzir o tecido gengival excessivo, expor as coroas clínicas desejáveis e corrigir alterações relacionadas ao contorno gengival, podendo ser realizada através de diferentes técnicas (BENNANI V, et al., 2017). Previamente a tal procedimento, alguns aspectos estéticos do sorriso devem se ser considerados para serem objetivamente atingidos, dentre eles a linha do sorriso, o zênite gengival, o contorno gengival, a margem gengival, a presença de papila interdental e a proporção larguraaltura dentária. Quando um ou mais destes fatores se apresentam de forma irregular, podem afetar significativamente a aparência dos dentes naturais e protéticos (BENNANI V, et al., 2017; BATRA P, et al.,2018).

De acordo com Batra $P$, et al. (2018) as alterações do contorno gengival e, consequentemente, do zênite gengival demostraram menor impacto na estética do sorriso quando observados por leigos, diferente da variação da posição da papila interdental, a qual obteve o maior impacto negativo, gerando os conhecidos black triangle ou triângulos negros, seguido por mudanças de cor da gengiva de origem inflamatória ou pigmentação melânica. Apesar disso, ainda assim, o zênite gengival é um dos pontos mais importantes para a estética dos dentes anterossuperiores. O mesmo se refere ao ponto mais apical do contorno gengival de cada dente anterior, geralmente localizado levemente distalizado ao longo eixo do dente, com exceção para o incisivo lateral superior, cujo zênite é preconizado localizar-se centralmente ao longo eixo do dente. A união dos zênites dos dentes incisivos superiores centrais e laterais e caninos é responsável por formar a linha gengival ou linha cervical e está diretamente ligada ao contorno gengival (TRUSHKOWSKY R, et al., 2016; BENNANI V, et al., 2017). Na apresentação clínica final dos casos apresentados no presente estudo, nota-se a diferença que a correção do zênite faz. Como podemos observar no caso 1 em que a paciente apresentava um sorriso mais infantilizado e após o procedimento, percebe-se um sorriso mais harmônico. E no caso 2 em que era totalmente desproporcional devido à hiperplasia e após o procedimento, o sorriso se mostra mais estético.

Quanto à escolha da técnica de gengivectomia a ser realizada, vários aspectos devem ser levados em consideração. Dentre eles, o fenótipo periodontal. Das variadas classificações de fenótipo periodontal, a de De Rouck T, et al. (2009) é a mais utilizada, na qual o mesmo pode ser classificado em 03 grupos: finofestonado, em que a gengiva se mostra fina e clara, geralmente encontrada no sexo feminino, plano-espesso, uma gengiva espessa e clara, encontrada no sexo masculino, e espesso-festonado. Junto a isso, o estudo de Arora R, et al. (2013) mensurou o crescimento tecidual após ACC em cada um dos fenótipos periodontais, relacionando-o à necessidade de se preconizar uma região para acomodação da inserção conjuntiva, em que foi observado que no fenótipo fino-festonado, a mesma é de aproximadamente $2 \mathrm{~mm}$, espesso-festonado $3 \mathrm{~mm}$ e plano-espesso $4 \mathrm{~mm}$. Desta forma, é de suma importância considerar o fenótipo periodontal quanto à quantidade de osso que deve ou não ser removida para propiciar a correta e devida acomodação da inserção conjuntiva.

O espaço biológico, atualmente denominado de inserção tecidual supracrestal, é composto pelo epitélio juncional e inserção conjuntiva. A primeira estrutura refere-se à adesão do tecido gengival ao esmalte dentário, sendo uma adesão primária e facilmente rompida. Já o segundo segmento refere-se à adesão direta de fibras colágenas do tecido gengival ao cemento radicular, constituindo-se como uma adesão firme (CARVALHO CV, et al., 2016). Com relação, portanto, à inserção conjuntiva, é notória a sua importância de manutenção, considerando a barreira física que a mesma forma contra a invasão de bactérias ao periodonto de sustentação localizado logo apicalmente. Quanto à sua dimensão, a literatura tem preconizado ser de, pelo menos, $1 \mathrm{~mm}$, de forma variável entre os indivíduos. Dada sua importância para manutenção da saúde periodontal, em casos onde naturalmente não haja espaço para acomodação da inserção conjuntiva, ocasionando o posicionamento mais coronário da margem gengival que o habitual e um tipo de erupção dental passiva alterada, há a necessidade de realizar-se osteotomia com finalidade de repor o referido espaço (CAIRO F, et al., 2012; MELE M, et al., 2018). Os dois casos clínicos em questão, apesar da excessiva exposição de gengiva ao sorrir, durante exame clínico de sondagem transulcular e ao exame radiográfico, não se observou necessidade de osteotomia para acomodação da inserção conjuntiva, uma vez que a 
distância da junção cemento esmalte à crista óssea alveolar observada foi de 2 a $3 \mathrm{~mm}$ para o caso clínico 1 , enquanto que no caso clínico 2 havia uma situação de hiperplasia do tecido gengival de causa desconhecida até o presente momento.

A erupção passiva alterada é uma alteração encontrada em adultos e ocorre quando a margem gengival se encontra incisalmente localizada à convexidade cervical ou à junção cemento esmalte (JCE), e foi classificada há décadas por Coslet e colaboradores (1977) com base na relação entre a gengiva e a coroa clínica e a relação entre a junção cemento e a crista do osso do outro. Assim, foi classificada em Tipo 1, quando junção mucogengival encontra-se apicalmente distante à JCE e à crista óssea, apresentando, portanto, uma grande satisfatória de mais de $3 \mathrm{~mm}$ de gengiva queratinizada, porém com o aspecto clínico de coroas clínicas curtas, e em tipo 2, quando junção mucogengival é posicionada nas proximidades da JCE e, consequentemente, a dimensão da gengiva queratinizada podem não estarem satisfatórias. Além disso, os tipos 1 e 2 têm subcategorias $A$ e $B$. No subgrupo $A$, a crista óssea está localizada 1,5 a $2 \mathrm{~mm}$ apicalmente à JCE, enquanto que no subgrupo $B$, a crista óssea é encontrada diretamente adjacente ao nível da JCE, sendo, neste último caso, um espaço insuficiente para acomodação da inserção conjuntiva (COSLET JG, et al., 1977; MELE M, et al., 2018). Em relação ao caso clínico 1 do presente trabalho, a erupção passiva alterada observada pôde ser classificada no Tipo $1 \mathrm{~A}$, de acordo com a referida classificação, uma vez que, embora a paciente apresentasse coroas clínicas curtas, a distância da JCE à crista óssea suficiente para acomodação da inserção conjuntiva, e recorremos, então à técnica de gengivectomia sem a necessidade de osteotomia (COSLET JG, et al., 1977).

Com relação ao caso clínico 2 de hiperplasia gengival do presente trabalho, sabe-se que esta condição clínica de etiologia multivariada é caracterizada pelo aumento progressivo da gengiva, causado por um aumento nos elementos do tecido conjuntivo submucoso, que pode causar problemas estéticos e/ou funcionais. A etiologia e patogênese da hiperplasia gengival ainda não estão bem estabelecidas, mas podem estar diretamente ligadas a três fatores: suscetibilidade individual, fatores locais (placa dentária, cárie e fatores iatrogênicos) e a ação de substâncias químicas e seus metabólitos (SHETTY AK, et al., 2010).

O paciente referido no caso clínico 2 não apresentava histórico prévio de uso de aparelho ortodôntico ou medicação que poderia ter causado tal alteração. Logo, acredita-se que sua etiologia possa ser relacionada a fatores genéticos inerentes ao próprio paciente. Assim, conforme preconizado na literatura científica, tal remoção do tecido gengival ocorreu através de incisão em bisel externo, excluindo-se as bolsas periodontais falsas. A osteotomia também foi descartada. Após o bisel externo, incisões horizontais e intrasulculares no colar gengival, o mesmo foi retirado e realizou-se o peeling gengival para afinamento do tecido. Essa técnica tem, como desvantagem, a exposição de tecido conjuntivo, porém possibilita renovação epitelial e afinamento do tecido gengival (KATHARIYA R; PRADEEP AR, 2011; MONEIM RAA, et al., 2017). Assim, para a realização da gengivectomia vários fatores devem ser analisados previamente ao procedimento, entre eles fatores estéticos como o zênite, a linha do sorriso além do biótipo periodontal, a causa da alteração, a necessidade ou não de osteotomia entre outros fatores.

\section{REFERÊNCIAS}

1. ARORA R, et al. Supracrestal Gingival Tissue: Assessing Relation with Periodontal Biotypes in a Healthy Periodontium. The International Journal of Periodontics \& Restorative Dentistry, 2013; 33(6): 763-771.

2. BATRA $P$, et al. Impact of altered gingival characteristics on smile esthetics: Laypersons' perspectives by $Q$ sort methodology. American Journal of Orthodontics and Dentofacial Orthopedics, 2018; 154(1): 82-90.

3. BENNANI V, et al. The periodontal restorative interface: esthetic considerations. Periodontology 2000, 2017; 74(1): 74-101.

4. CAIRO F, et al. Periodontal plastic surgery to improve aesthetics in patients with altered passive eruption/gummy smile: a case series study. International Journal of Dentistry. 2012; 2012:837658.

5. CARVALHO CV, et al. Espaço Biológico: Conceito Chave para Estética e Saúde Gengival em Procedimentos Restauradores. The International Journal of Periodontics and Restorative Dentistry, 2016; 1(1): 20.

6. COSLET JG, VANARSDALL R, WEISGOLD A. Diagnosis and classification of delayed passive eruption of the dentogingival junction in the adult. The Alpha Omegan. 1977; 70(3):24-8.

7. DE ROUCK T, et al. The gingival biotype revisited : transparency of the periodontal probe through the gingival margin 
as a method to discriminate thin from thick gingiva. Journal of Clinical Periodontology, 2009; 36(5): 428-433.

8. FALABELLA MEV, et al. Crown lengthening - Case reports. Brazilian Journal Periodontology, 2015; 25(2): 55-59.

9. KALSI HJ, et al. An Update on Crown Lengthening. Part 2: Increasing Clinical Crown Height to Facilitate Predictable Restorations. Dental Update, 2015; 42(3): 230-236.

10. KATHARIYA R, PRADEEP AR. SPLIT mouth de-epithelization techniques for gingival depigmentation: A case series and review of literature. Journal of Indian Society Periodontology, 2011; 15(2):161-8.

11. MALKINSON S, et al. The Effect of Esthetic Crown Lengthening on Perceptions of a Patient's Attractiveness, Friendliness, Trustworthiness, Intelligence, and Self-Confidence. Journal of Periodontology, 2013; 84(8): 1126-1133.

12. MELE M, et al. Esthetic treatment of altered passive eruption. Periodontology 2000, 2018; 0(27): 1-19.

13. MONEIM RAA, et al. Gingival pigmentation (cause, treatment and histological preview ). Future Dental Journal, 2017 ; $3(1): 1-7$.

14. RIBEIRO FV, et al. Open-Flap Versus Flapless Esthetic Crown Lengthening: 12-Month Clinical Outcomes of a Randomized Controlled Clinical Trial. Journal of Periodontology, 2014; 85(4): 536-544.

15. SEIXAS MR, et al. Checklist dos aspectos estéticos a serem considerados no diagnóstico e tratamento do sorriso gengival. Dental Press Journal of Orthodontics, 2011; 16(2): 131-157.

16. SHAHABE SA, et al. Esthetic Crown Lengthening: Theoretical Concepts and Clinical Procedures. International Journal of Contemporary Dentistry, 2012; 3(3): 33-38.

17. SHETTY AK, et al. Idiopathic gingival enlargement and its management. Journal of Indian Society of Periodontology, 2010; 14(4): 263-265.

18. SILVA CO, et al. Aesthetic crown lengthening: Periodontal and patient-centred outcomes. Journal of Clinical Periodontology, 2015; 42(12): 1126-1134.

19. SMITH MM. Gingivectomy, gingivoplasty, and osteoplasty for mandibular canine tooth malocclusion. Journal of Veterinary Dentistry, 2013; 30(3): 184-197.

20. TRUSHKOWSKY R, et al. Digital Smile Design concept delineates the final potential result of crown lengthening and porcelain veneers to correct a gummy smile. The International Journal of Esthetic Dentistry, 2016; 11(3): 338-354,.

21. YADAV SR, et al. Altered Passive Eruption : Report on Management of Two Cases Altered Passive Eruption : Report on Management of Two Cases. Journal of Contemporary Dentistry, 2015; 5(3): 173-177. 